\title{
Cambio climático y recursos hídricos en Colombia
}

\section{Climate change and water resources in Colombia}

\author{
Juan Alarcón-Hincapié ; Carlos Zafra-Mejía; Lena Echeverri-Prieto ${ }^{3}$
}

11.ng. Catastral y Geodesta, M.Sc., Ph.D., Universidad Distrital Francisco José de Caldas, Facultad del medio Ambiente y Recursos Naturales. Bogotá D.C., Colombia, e-mail:jcalarconh@udistrital.edu.co; Dhttps://orcid.org/0000-0003-0872-6116

${ }^{2}$ Ing. Civil, M.Sc. Ingeniería Ambiental, Ph.D. Universidad Distrital Francisco José de Caldas, Facultad del medio Ambiente y Recursos Naturales, Bogotá D.C., Colombia, e-mail: czafra@udistrital.edu.co; Dhttps://orcid.org/0000-0002-4061-4897

${ }^{3}$ Microbióloga, M.Sc. Universidad Distrital Francisco José de Caldas, Facultad del medio Ambiente y Recursos Naturales. Bogotá D.C., Colombia, e-mail:Icecheverryp@udistrital.edu.co; Dhttps://orcid.org/0000-0002-6940-4383

Cómo citar: Alarcón-Hincapié, J.; Zafra-Mejía, C.; Echeverri-Prieto, L. 2019. Cambio climático y recursos hídricos en Colombia. Rev. U.D.C.A Act. \& Div. Cient. 22(2):e1368. http://doi.org/10.31910/rudca.v22.n2.2019.1368

Artículo de acceso abierto publicado por Revista U.D.C.A Actualidad \& Divulgación Científica, bajo una licencia Creative Commons CC BY-NC 4.0

Recibido: Julio 17 de 2018

Aceptado: Septiembre 22 de 2019

Editado por: Ingeborg Zenner de Polanía

\section{RESUMEN}

La presente investigación tuvo por objeto evaluar los posibles impactos que habría en la distribución espacial del recurso hídrico sobre el territorio colombiano, bajo dos escenarios de cambio climático, a mediados del siglo XXI. Para el efecto, se utilizaron dos indicadores hídricos ambientales (Índice de Aridez y Balance Hídrico), con los cuales, se estima y se cuantifica la oferta hídrica del territorio colombiano, a partir de datos climatológicos del período de referencia 1976-2005 y, posteriormente, utilizando los datos de las variables climatológicas de los escenarios RCP 4,5 y 6,0, de la tercera comunicación nacional de Colombia ante la convención marco de cambio climático. Los resultados de esta modelación arrojaron los siguientes resultados: el régimen hidrológico del país, se caracteriza por tener una escorrentía promedio de $1.644 \mathrm{~mm}$, con valores que van desde una escorrentía promedio de $100 \mathrm{~mm}$ al año, en la península de la Guajira, hasta escorrentías mayores de $6.000 \mathrm{~mm}$, en el Pacífico. El índice de aridez modelado arrojó un valor promedio de 0,23 , ubicando a Colombia en condiciones Moderadas y Excedentes de agua. Se presentarían reducciones significativas en el volumen de escorrentía hasta en un $18 \%$ en promedio para los dos escenarios. La menor reducción se daría en el escenario RCP 4,5, con un $12 \%$ de la escorrentía actual y la reducción más drástica, se presentaría en el RCP 6,0, hasta en un $18 \%$. En cuanto al Índice de Aridez, se sugiere que el escenario más fuerte es el RCP 6,0, con un valor promedio de 0,50 lo que significa una mderada situación deficitaria de agua.

Palabras clave: cambio climático; recursos hídricos; balance hídrico; índice de aridez

\section{ABSTRACT}

The possible impacts from the spatial distribution of the water resource over the Colombian territory are analyzed under two scenarios of climate change in the middle of the 21 st century. For the effect, two environmental water indicators were used (Aridity index and Water Balance) to estimate, quantify and water supply of the Colombian territory, from the climate data during the period of reference 1976-2005 in spatial resolution of 900 meters. In this 
modeling, the water regimen of the country is characterized by its average runoff of $1644 \mathrm{~mm}$, with values that are between an annual average runoff of $100 \mathrm{~mm}$ in the Guajira peninsula until higher runoffs of 6000 millimeters in the Pacific. The aridity index in such modeling generated an average of 0.37 , putting Colombia in moderate conditions of aridity, which shows sectors with a high-water deficit such as the Guajira peninsula and in the north of Magdalena and Atlántico regions. Additionally, the climate variables for Colombia were taken by a regional climate modeling in two scenarios RCP 4.5 and 6.0 for the period 2011-2040 and the environmental water indicators were generated. Across a comparison between the indicators of the current (1976-2005) and future period 2011-2040, the changes that would appear in the water availability towards those periods in both scenarios were established. The results are summarized in: substantial reductions in the runoff volume in respect to the current values in an $18 \%$ average for all scenarios, the lowest reduction would appear in 4,5 with $12 \%$ of the current runoff and the most drastic reduction presents in 6,0 in the middle of the century until $18 \%$. Regarding the aridity index, the most aggressive scenario is 6.0 with an average value of 0,50 , which refers to a water deficit, while by the middle of the century 6,0 and 4,5 would locate the country in a moderate condition of water deficit.

Keywords: climate change; water resources; water balance; aridity index.

\section{INTRODUCCIÓN}

Tal como se ha previsto, las alteraciones originadas por el Cambio Climático impactarán las diferentes regiones del mundo (IPCC, 2016). En efecto, el cambio climático, expresado en la modificación de la temperatura media, la precipitación anual, la humedad relativa y la escorrentía, posibilitarán transformaciones para el ciclo hidrológico y, por ende, se hará más compleja la planificación de los recursos hídricos, por tanto, se tendrán efectos importantes sobres los recursos de agua, a escala regional (Menzel \& Burger, 2002; Muzik, 2002), por lo que resulta fundamental llevar a cabo un adecuado manejo, gestión y planificación del mismo. Existen modelos hidrológicos que permiten estimar los valores de escorrentía actual a escala de grandes extensiones, a partir de las variables precipitación y temperatura, simuladas por los modelos regionales de clima (MRC). Entre ellos, se pueden mencionar el modelo de Yates (1997); WBM (Water Balance Model), de Vörösmarty et al. (1998); WaterGAP (Water-Global Assessment and Prognosis), de Döll et al. (1999) y SIMPA (Sistema Integrado de Modelización Precipitación Aportación) (Estrela et al. 1996). Por otro lado, se han planteado modelos de balances hídricos mensuales, a nivel de escala macro y semi-distribuido, que han sido desarrollados para simular y predecir los procesos hidrológicos, utilizando herramientas de Información Geográfica (Guo et al. 2002). Se han llevado a cabo varios estudios relacionados con la gestión de los recursos hídricos, debido al déficit de agua en las diferentes regiones (Agrawala \& Fankhauser, 2008; Tian et al. 2008; Yang et al. 2008; Hendra et al. 2013).

El cambio climático representa uno de los mayores desafíos que deberá enfrentar la humanidad durante este siglo. Magrin et al.
(2007) plantean que los efectos de este fenómeno se podrían intensificar en el futuro, en caso de que no se extiendan a escala regional y global, las acciones necesarias para mitigar las emisiones de gases de efecto invernadero y no se instrumenten las medidas e inversiones correspondientes para la adaptación a las nuevas condiciones climáticas.

Las simulaciones del clima futuro, según el IPCC (2016), se han efectuado con base en escenarios socio-económicos diseñados previamente, que caracterizan las posibles variaciones futuras en la concentración de gases de efecto invernadero, debido a cambios en la sociedad, en la economía y en las fuentes de energía. Para el Quinto Informe de Evaluación del Grupo Intergubernamental de Expertos sobre el Cambio Climático (AR5), la comunidad científica ha definido un grupo nuevo de escenarios, denominados "trayectorias de concentración representativas" RCP, por sus siglas en idioma inglés. Estos escenarios, se centran en las emisiones antropogénicas y no incluyen cambios en impulsores naturales, como el forzamiento solar o volcánico o las emisiones naturales de $\mathrm{CH}_{4} \mathrm{O} \mathrm{N} \mathrm{N}_{2} \mathrm{O}$. Los RCP representan el forzamiento radiactivo total calculado para el 2100 respecto a 1750 . Se podría decir que el RCP 2,6 representa un escenario de mitigación, el RCP 4,5 y RCP 6,0 son escenarios de estabilización y el RCP 8,5 corresponde a un escenario con un nivel muy alto de emisiones de gases de efecto invernadero. La presente investigación tuvo por objeto evaluar el posible impacto de cambio climático en el recurso hídrico del territorio colombiano, bajo dos escenarios de cambio climático (RCP 4,5 y RCP 6,0), para el periodo 2040.

\section{MATERIALES Y MÉTODOS}

El estudio, se realizó en dos fases, a saber: 1) La modelación espacial actual de la oferta hídrica natural disponible y de sus indicadores asociados en Colombia, con una base climática 1976-2005 (IDEAM et al. 2005) y, 2) la elaboración de mapas de las posibles distribuciones futuras de la oferta hídrica natural disponible, considerando el periodo entre 2011-2040, bajo los escenarios de cambio climático RCP 4,5 y 6,0, por IPCC (2016). Para lo anterior, se utilizó el software ArcGis 10.4 y el método de interpolación empleado fue el inverso de la distancia ponderada (IDW) de propósito nominal, el cual, estima valores de las celdas vecinas a partir de valores de entrada. Para el presente proyecto de investigación, se propuso una distancia de $900 \mathrm{~m}$ entre cada celda. Esta metodología requiere que las estaciones estén cercanas, ya que, a mayor cercanía, menor incertidumbre. Además, es el método con el que se obtiene la menor diferencia entre valores registrados y los estimados, de acuerdo con una prueba de comparación de metodologías de interpolación, realizado por Drummond et al. (2007).

Posteriormente, se determinó el valor promedio de la escorrentía superficial e índice de aridez futuros y se compararon con los rangos propuestos en el Estudio Nacional del Agua, año 2014.

La modelación espacial actual régimen hídrico (Índice de aridez) y de la oferta hídrica superficial (Escorrentía) para Colombia. La caracterización y la estimación de la oferta hídrica 
superficial, se soportan en los procesos del ciclo del agua y en la cuantificación de sus componentes, a partir del balance hídrico, en particular, el de escorrentía y su expresión en términos de rendimiento hídrico.

Ciclo hidrológico. Según UNESCO (2010), se define como la "Sucesión de fases por las que pasa el agua en su movimiento de la atmósfera a la tierra y en su retorno a la misma: evaporación del agua del suelo, mar y aguas continentales, condensación del agua en forma de nubes, precipitación, acumulación en el suelo o en masas de agua y reevaporación". Los componentes del ciclo hidrológico que se relacionan más directamente con la disponibilidad de agua y que intervienen en la ecuación del balance hídrico son la precipitación, como variable de entrada; la evapotranspiración real y la escorrentía, como variables de salida.

La precipitación es el volumen de agua que cae por acción de la gravedad sobre la superficie terrestre en forma de lluvia, llovizna, nieve o granizo, procedentes de la condensación del vapor de agua (ENA, 2014). La evapotranspiración real (ETR), sinónimo de evapotranspiración efectiva, se define como la suma de las cantidades de agua evaporadas del suelo y de las plantas cuando el terreno se encuentra con su contenido natural de humedad. Se diferencia de la evapotranspiración potencial (ETP), que es la cantidad máxima de agua capaz de ser evaporada en una condición climática determinada, con una cubierta vegetal continua y suficiente disponibilidad de agua (UNESCO, 2010). La escorrentía hídrica superficial (ESC) o flujo superficial, se define como parte de la precipitación que fluye por la superficie del suelo y se concentra en los cauces y los cuerpos de agua. Es la lámina de agua que circula sobre la superficie en una cuenca de drenaje. En el balance hídrico, se considera como la precipitación menos la evapotranspiración real y la infiltración en suelo está en función de las características topográficas, geológicas, climáticas y de vegetación y está íntimamente ligada a la relación entre aguas superficiales y subterráneas de la cuenca (ENA, 2014).

Balance hídrico. Se basa en la ley física de conservación de masas y, según la UNESCO (2010), es definido como "Balance de agua basado en el principio de que durante un cierto intervalo de tiempo el aporte total a una cuenca o masa de agua debe ser igual a la salida total de agua más la variación neta en el almacenamiento de dicha cuenca o masa de agua". La formulación matemática del balance, por lo tanto, expresa la igualdad entre los aportes de agua que entran a un sistema hidrográfico determinado y la cantidad de agua que sale del sistema, considerando las variaciones internas en el almacenamiento de humedad ocurridas durante un periodo determinado.

$\mathrm{ESC}=\mathrm{P}-\mathrm{ETR}$

ecuación 1

Donde: ESC, Escorrentía hídrica superficial (mm); P, Precipitación (mm); ETR, Evapotranspiración real (mm), calculado así:

$\mathrm{ETR}=\mathrm{P} /\left(0.9+\left(\mathrm{P}^{2} / \mathrm{L}^{2}\right)\right)^{0.5}$

ecuación 2

$\mathrm{L}=300+25^{*} \mathrm{~T}+0.005 \mathrm{~T}^{3}$
Donde: ETR, evapotranspiración real (mm), según Turc (1954); ETP, evapotranspiración potencial $(\mathrm{mm})$; P, precipitación $(\mathrm{mm}) ; \mathrm{L}$, factor heliotérmico; T, temperatura $\left({ }^{\circ} \mathrm{C}\right)$

Índice de aridez (IA). Se ha sido definido como una característica cualitativa del clima, que permite medir el grado de suficiencia o insuficiencia de la precipitación para el sostenimiento de los ecosistemas de una región. Identifica áreas deficitarias o de excedentes de agua, calculadas a partir del balance hídrico superficial. La evapotranspiración potencial representa, para este caso, un factor determinante en la obtención de este índice. Para generar el indicador, se utilizan las ecuaciones de Turc, para el cálculo de la evapotranspiración real (ETR) y Thornwaite ajustada por brillo solar (latitud), para el cálculo de la evapotranspiración potencial (ETP). Este índice representa la dinámica superficial del suelo determinada a partir de las variables de evapotranspiración potencial (ETP) y de evapotranspiración real (ETR) y no se refiere a la dinámica subsuperficial del suelo, utilizada en análisis climáticos, para clasificar el grado de humedad del suelo, a través de la relación de la precipitación y la evapotranspiración potencial; para el cálculo, se utilizó la siguiente ecuación:

$\mathrm{Ia}_{\mathrm{a}}=\left(\mathrm{ETP}_{\text {ajustada }}-\mathrm{ETR}\right) / \mathrm{ETP}_{\text {ajustada }}$

ecuación 4

Donde: Ia, índice de aridez (adimensional); ETP ajustada, evapotranspiración potencial ajustada por brillo solar (mm); ETR, evapotranspiración real $(\mathrm{mm})$. Este índice representa la dinámica superficial del suelo, a partir de las variables de evapotranspiración potencial (ETP) y de evapotranspiración real (ETR) y no se refiere a la dinámica subsuperficial del suelo, utilizada en análisis climáticos, para clasificar el grado de humedad del suelo, a través de la relación de la precipitación y la evapotranspiración potencial. Calculado así:

$\mathrm{ETP}=12 * 16 *(10 * \mathrm{~T} / \mathrm{I})^{\mathrm{a}}$

ecuación 5

$\mathrm{I}=\left((\mathrm{T} / 5)^{1.514}\right) * 12$

ecuación 6

$\mathrm{a}=0.000000675 * \mathrm{I}^{3}-0.0000771 * \mathrm{I}^{2}+0.0179 * \mathrm{I}+0.49239$

ecuación 7

Donde: I, coeficiente anual; $\mathrm{T}$, temperatura promedio anual; a, coeficiente en la fórmula de Thornwaite.

Para este estudio, la ETP se ajustó por brillo solar (latitud), a partir de la siguiente ecuación:

$\mathrm{ETP}_{\text {ajustada }}=\mathrm{ETP}^{*} 1.02147$

ecuación 8

La temperatura media anual del aire, se obtuvo a partir de las mediciones en estaciones climatológicas del Instituto de Hidrología, Meteorología y Estudios Ambientales (IDEAM) y estimaciones con base en el Modelo Digital de Elevación (DEM) (Alarcón \& Pabón, 2013). La precipitación, se logró utilizando los datos de estaciones climatológicas suministradas por el IDEAM, complementadas a partir de la base de datos de la Tropical Rainfall Meassurement Mission (TRMM) 
La modelación espacial futura del régimen hídrico y de la oferta hídrica superficial para Colombia. La estimación de la oferta hídrica superficial futura, a partir del balance hídrico, en particular, el de escorrentía y el Índice de aridez, se simularon mediante la incorporación al modelo de los datos climatológicos de los escenarios propuestos, con base en la aplicación de un modelo climático regional para los periodos 2011-2040, bajo los escenarios de cambio climático RCP 4,5 y 6,0, por IPCC (2016). La distribución espacial de la temperatura media anual y la precipitación anual en esos escenarios, se interpoló utilizando IDW (Inverso de la Distancia Ponderada), para generar información a mayor resolución espacial (900m) y disponer de datos para el todo el territorio nacional y estandarizar la información del período 1976-2005.

\section{RESULTADOS Y DISCUSIÓN}

Temperatura, precipitación actual. Con base en la información climática básica, se generaron las capas de precipitación para el periodo 1976-2005; los valores mínimos y máximos reportados para la capa de precipitación fueron entre $242 \mathrm{~mm}$ y $1.4665 \mathrm{~mm}$, en la Guajira y el sector norte del departamento del Cauca, respectivamente. El mapa de temperatura media anual del aire es el resultado de la unión espacial de cada región climática, así el análisis consideró, de manera fidedigna, la distribución espacial de la temperatura, por tener en cuenta factores de tipo geográfico, como la altura sobre el nivel de mar, las variaciones locales por efecto la topografía y por el efecto de barlovento y sotavento. En cuanto a la humedad relativa los valores mínimos, se dan en los límites de los departamentos del Tolima y Huila y los departamentos de la Guajira y Norte de Santander; los valores extremos, se presentan en el Pacífico y sur del país, con valores entre el 85 y 95\%; el resto del país, se caracteriza por humedades relativas ente el 75 y $85 \%$ (Alarcón \& Pabón, 2013) (Figura 1).

La estimación del régimen hídrico y de la oferta hídrica superficial actual para Colombia.

Escorrentía. El régimen hidrológico del país, se caracteriza por tener una escorrentía promedio de $1.644 \mathrm{~mm}$, con valores que van desde una escorrentía promedio de $100 \mathrm{~mm}$ al año, en la península de la Guajira, hasta escorrentías mayores de 6.000 milímetros, en el Pacífico. En el Caribe, con excepción de la Sierra Nevada de Santa Marta y el sector norte del departamento de Antioquia, en la cuenca del río Atrato, presenta valores con escorrentías bajas, no superando los $400 \mathrm{~mm}$, mientras que otras regiones, como el Pacífico, en particular, el sur del departamento del Chocó y occidente de los departamentos del Cauca y Valle del Cauca, específicamente, en la cuenca alta del río San Juan, se presentan escorrentías muy altas, mayores de $5.000 \mathrm{~mm}$.
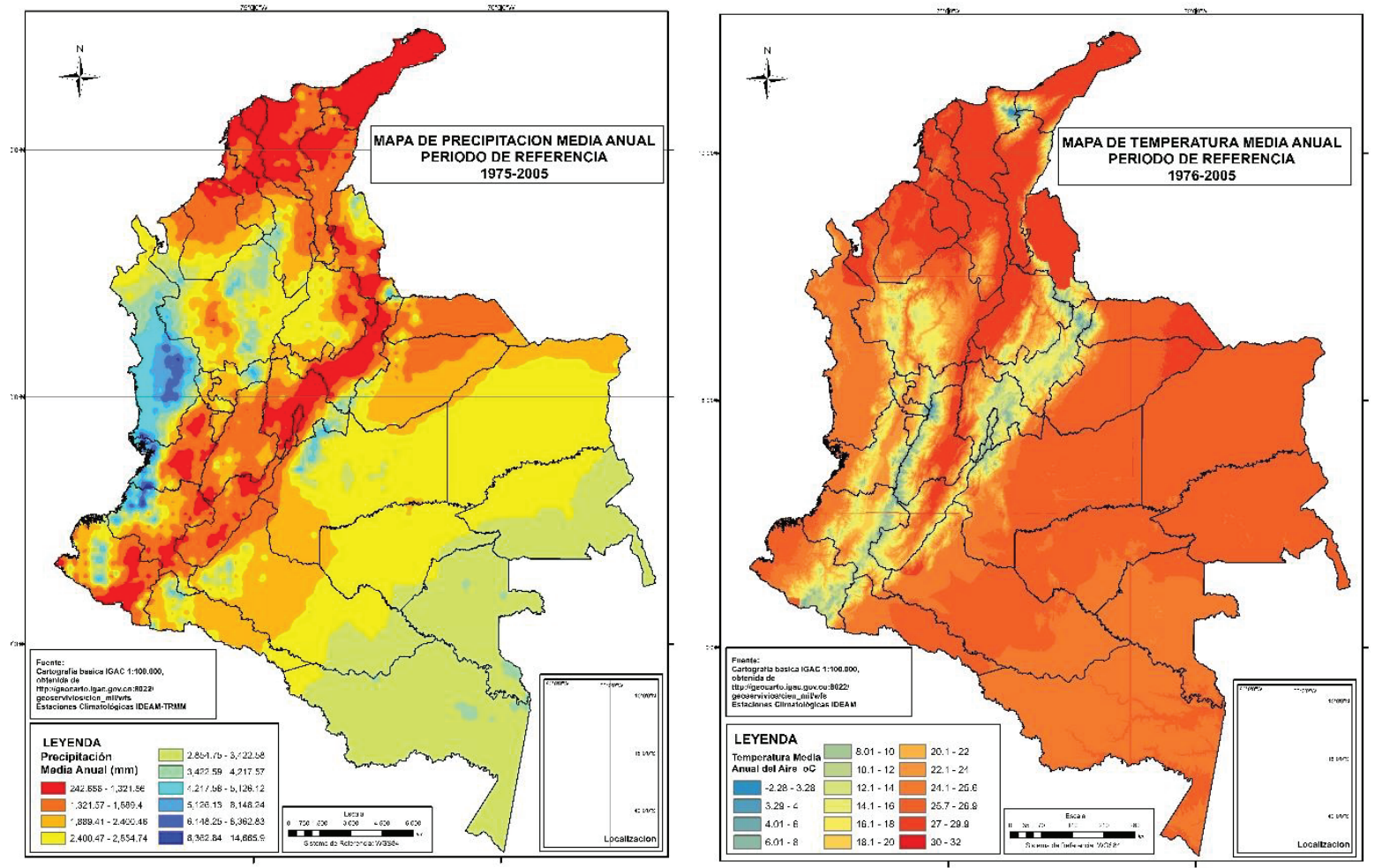

Figura 1. Mapa de precipitación media anual y temperatura media anual del aire periodo de referencia 1976-2005. 
En la figura 2, se muestran valores bajos de escorrentía en los Altiplanos cundiboyacense y nariñense, en el departamento del Valle del Cauca; la cuenca alta del río Patía, en el desierto de la Tatacoa, en el Huila; el cañón de la cuenca del río Chicamocha y la cuenca alta del Catatumbo, en Santander y Norte de Santander; sin embargo, para el resto de la región andina, la escorrentía se considera moderada, con valores entre $1.500 \mathrm{~mm}$ y $2.500 \mathrm{~mm}$, cubriendo el $31 \%$ del territorio nacional. El piedemonte llanero, se caracteriza por tener una escorrentía media alta, valores mayores de $2.000 \mathrm{~mm}$. En los departamentos del Arauca y Casanare, se presentan valores medios de escorrentía, hasta con $1.000 \mathrm{~mm}$; en general, la amazonia colombiana presenta escorrentías que van desde los 1.000 hasta los $3.000 \mathrm{~mm}$.

Índice de aridez. Las características del régimen hidrológico están determinadas por factores climatológicos y bióticos, asociados con la cobertura de la superficie terrestre y los propios de la dinámica del agua en el suelo y subsuelo. ENA (2014), en esta investigación, se utilizó como indicador para estudiar el régimen hidrológico el índice de aridez, cuyo valor promedio, para esta investigación, se calculó en 0,23 , ubicando a Colombia en condiciones moderadas y excedentes de agua; a su vez, presenta sectores altamente deficitarios de agua, localizados en la península de la Guajira y norte de los departamentos de Magdalena y Atlántico, así como también en los límites de los departamentos de Cundinamarca y Tolima y sur del departamento de Norte de Santander; las zonas deficitarias de agua ocupan un $6,9 \%$ del territorio colombiano y se localizan en la cuenca del río grande de la Magdalena, norte del departamento de Córdoba, Sucre, Magdalena, Cesar y parte del departamento de Norte de Santander; las condiciones moderadas a deficitarias de agua se dan en la sabana de Bogotá y valle del río Chicamocha, así como también en la Orinoquia y amazonia colombiana. Es importante mencionar que un porcentaje muy alto $(48,5 \%)$ del país presenta excedentes de agua superficial, de moderados a altos. Cabe resaltar que cerca del $80 \%$ de la población y de las actividades económicas del país están localizadas en cuencas con déficit natural de agua, ilustrados en la figura 2.
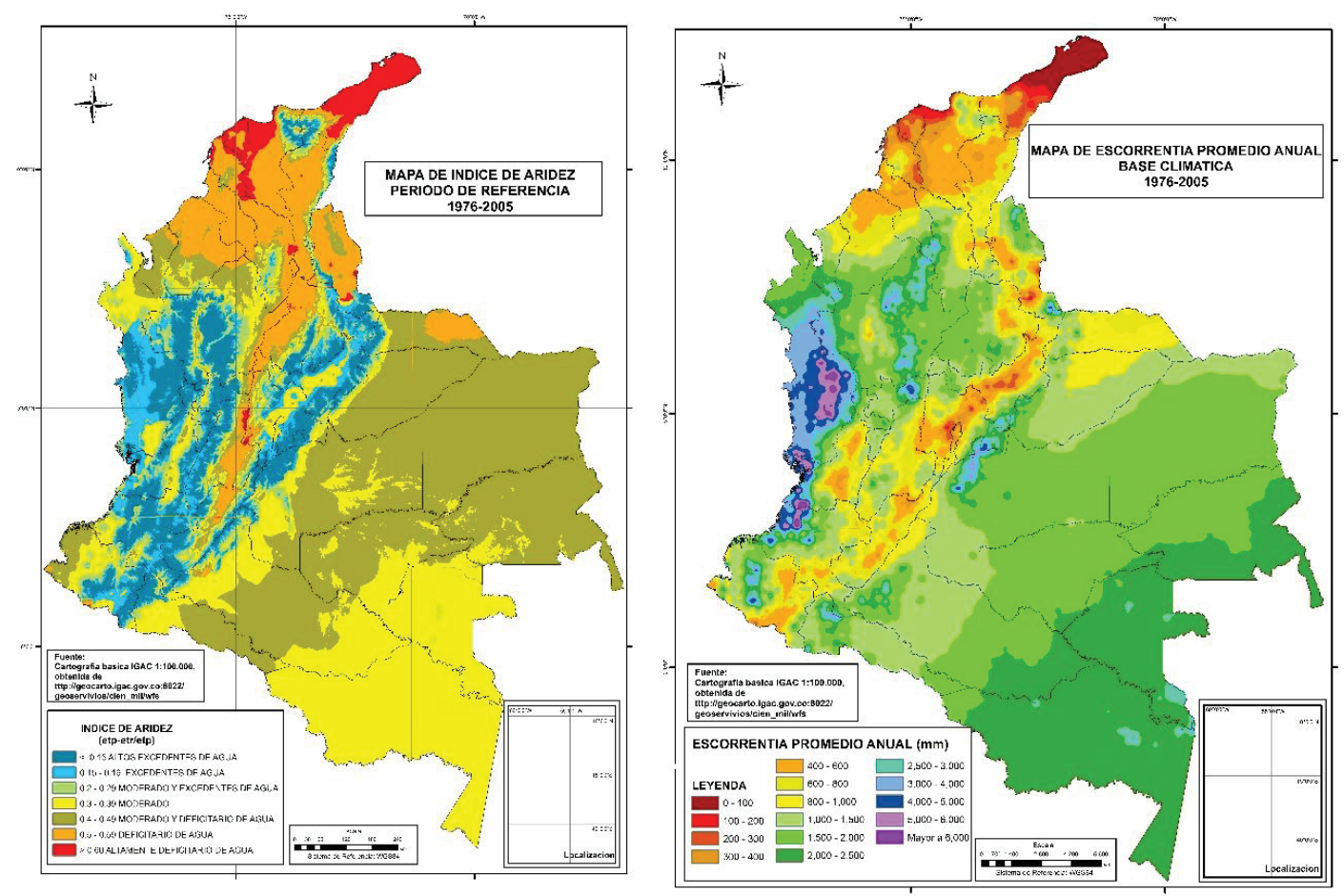

Figura 2. Mapa de escorrentía promedio anual (izquierda) e Índice de Aridez (derecha), periodo de referencia $1976-2005$.

Temperatura y precipitación futuras. En términos generales, para el período 2011-2040, la temperatura del aire aumentaría alrededor de los $2^{\circ} \mathrm{C}$ por encima de lo observado en el período de referencia, con aumentos de hasta $4^{\circ} \mathrm{C}$, en diferentes regiones, al finalizar el período. El calentamiento tiende a ser mayor en el Valle del Magdalena, en la Orinoquía, un extenso sector de la Amazonía y el sector central del litoral Caribe, así como en el sur de la costa Pacífica, principalmente, en el departamento de Nariño.
En los dos escenarios evaluados (4,5 y 6,0), la precipitación anual durante el período 2011-2040, tendría un incremento mayor del $10 \%$, comparada con la que se observaba en el período de referencia; estos aumentos se observan, principalmente, en las regiones de la Orinoquia y de la Amazonia, el piedemonte amazónico y llanero, el Alto y Medio Magdalena, todo el Altiplano cundiboyacense, nororiente del Chocó. Por otro lado, se aprecian núcleos con incrementos mayores, especialmente, en el piedemonte llanero, parte 
alta del río Arauca, norte del Chocó y un sector al sur este de la Sierra Nevada de Santa Marta. Reducción de la precipitación anual, en el período mencionado, se observaría en la cuenca del río Cauca, en el Pacífico Sur y un sector centro-sur del Chocó, así como una amplia zona en la región Caribe (Alarcón \& Pabón, 2013) (Figura 3).
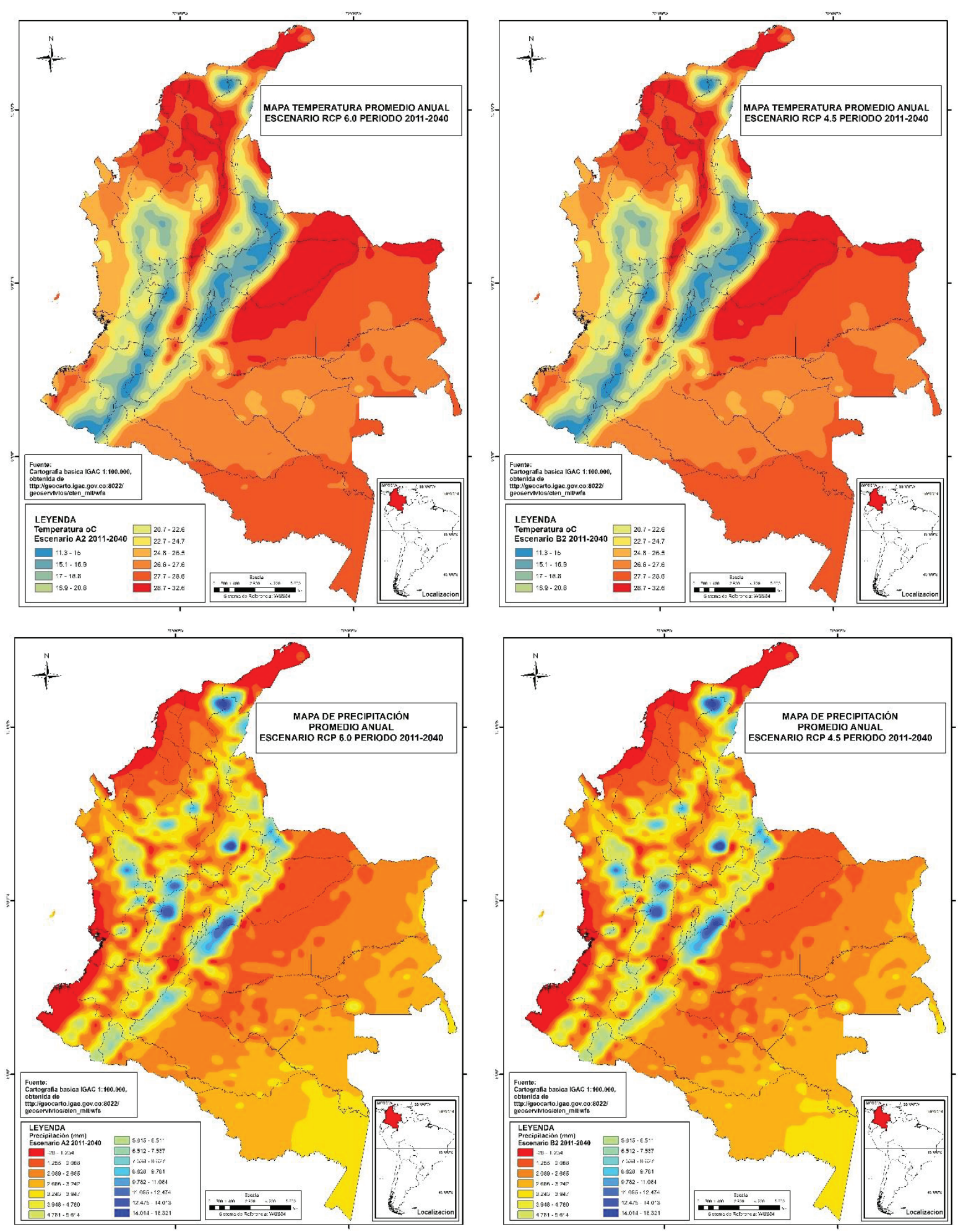

Figura 3. Temperatura media anual del aire (arriba) y precipitación anual (abajo), bajo los escenarios RCP 4,5 y 6,0, periodo $2011-2040$. 
Escorrentía futura. Al ejecutar el modelo para evaluar la distribución potencial de la escorrentía para los escenarios de cambio climático propuestos, se generaron dos mapas, con los dos indicadores para el periodo analizado y bajo los escenarios de cambio climático RCP 6,0 y 4,5 (Figura 4).
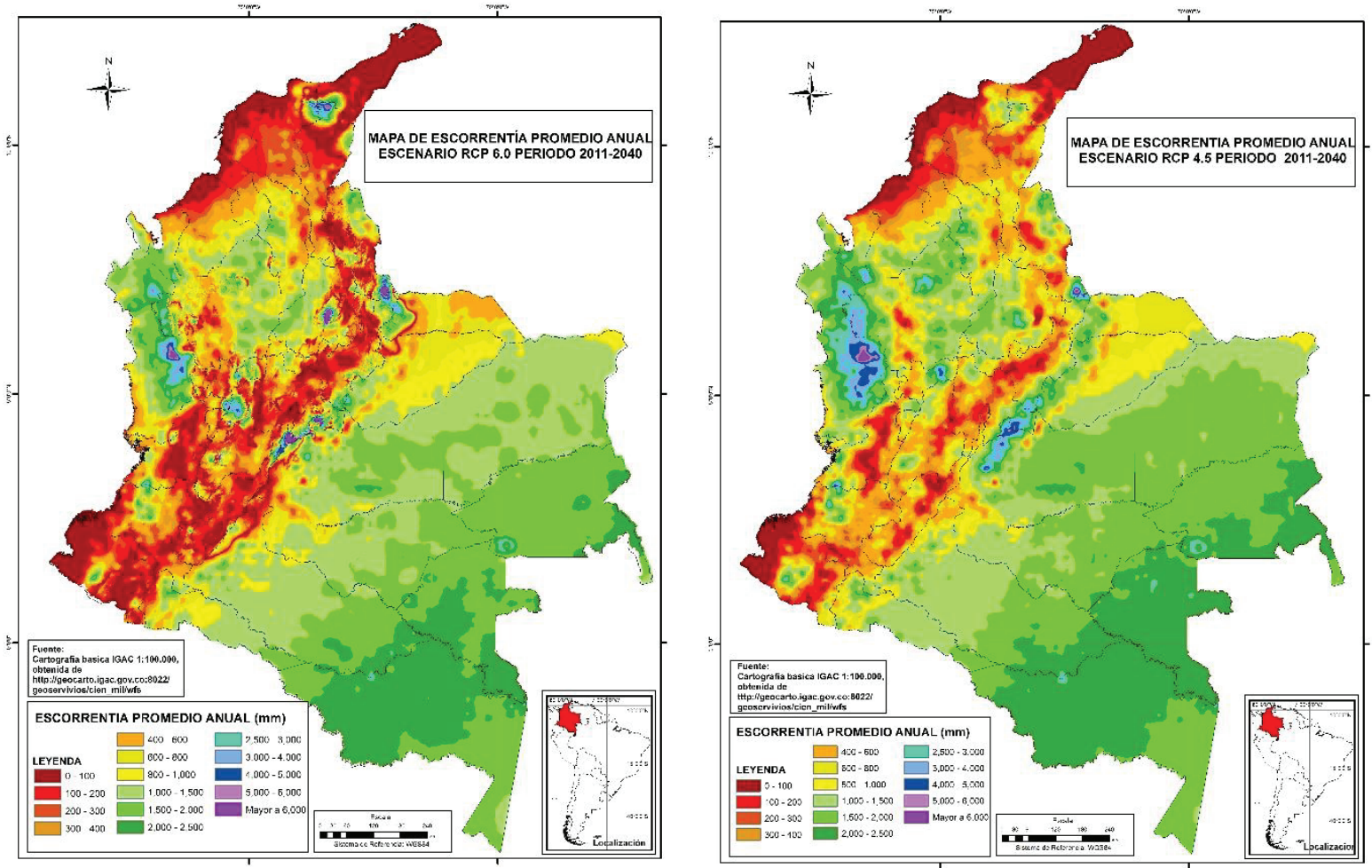

Figura 4. Distribución potencial de la escorrentía bajo los escenarios 6,0 y 4,5, periodo 2011-2040.

Se observa que el comportamiento de las nuevas áreas varía en función del escenario utilizado, generándose valores promedios de escorrentía así:

Escorrentía para Escenario RCP 4,5 $=1.439 \mathrm{~mm}$

Escorrentía para Escenario RCP 6,0 = 1.344mm

De los datos arrojados por el modelo podrían sugerir posibles reducciones significativas en el volumen de escorrentía con relación al valor actual en un $15 \%$. en promedio para todos los escenarios; la menor reducción se daría en el escenario 6,0 , con un $18 \%$, de la escorrentía actual y un $12 \%$, para el escenario 4,5 , respecto a la actualidad.

En la figura 4, se observa que los escenarios 4,5 y 6,0 en la modelación, estiman valores de escorrentía, con promedios muy bajos $(100 \mathrm{~mm})$, en el litoral norte a excepción de la Sierra Nevada de Santa Marta, así como también en el departamento de Nariño, un núcleo en el Valle del Cauca, en el departamento del Huila, límites entre Tolima y Cundinamarca y el Departamento de Boyacá. Otro núcleo bajo de escorrentía se presentaría en los departamentos de Arauca y Casanare. Se mostrarían valores de hasta $600 \mathrm{~mm}$ en gran parte de la cuenca Magdalena Cauca, excluyendo sectores de Antioquia y Santander, con valores de escorrentía que irían hasta los $3.000 \mathrm{~mm}$; los valores muy altos de escorrentía, se presentarían en el Choco y algunos sectores del piedemonte llanero, la amazonia y la Orinoquia, con valores entre los 1.000 y los $2.500 \mathrm{~mm}$. Para el escenario 6,0, se resaltan los promedios bajos de escorrentía, principalmente, para los departamentos de Nariño, Huila, Cauca, el Valle del Magdalena Cauca, casi el 90\% del departamento de Cundinamarca y Boyacá. La cuenca del río Chicamocha, norte de Santander y la Guajira, seguiría su condición de muy baja escorrentía, pero incrementando su superficie a regiones circundantes de la Sierra Nevada de Santa Marta. Otro aspecto importante de mencionar es que la modelación sugiere aumentos considerables de las regiones con escorrentía superior a los $6.000 \mathrm{~mm}$, localizándose, principalmente, en el sur del Departamento del Choco y Norte del Valle del Cauca; en general, la Amazonía y la Orinoquia presentarían cierta estabilidad en los escenarios modelados, con valores promedio entre los 1.000 y los $3.000 \mathrm{~mm}$.

Índice de aridez futuro. Al modelar el índice de aridez para los escenarios 4,5 y 6,0 , se sugiere que el escenario más fuerte es el 6,0 , pues pondría al país en un valor promedio de 0,50 , lo que significa una situación deficitaria de agua, mientras que el 4,5, pondría al país en una condición moderada y deficitaria de agua, como se aprecia en la figura 5. La modelación en los dos escenarios 
destaca que las regiones altamente deficitarias de agua en promedio ocuparían un 15\% del territorio nacional, localizándose en la costa norte colombiana, a excepción de la Sierra Nevada, en el valle del Magdalena y el sector occidental del departamento de Nariño y los departamentos de Arauca y Casanare; la zona Andina presentaría altos y moderados excedentes de agua; el norte de la región Pacífica presentaría condiciones moderadas a deficitarias de agua y la amazonia y Orinoquia, en general, cambiarían su condición a deficitaria de agua (Figura 5).
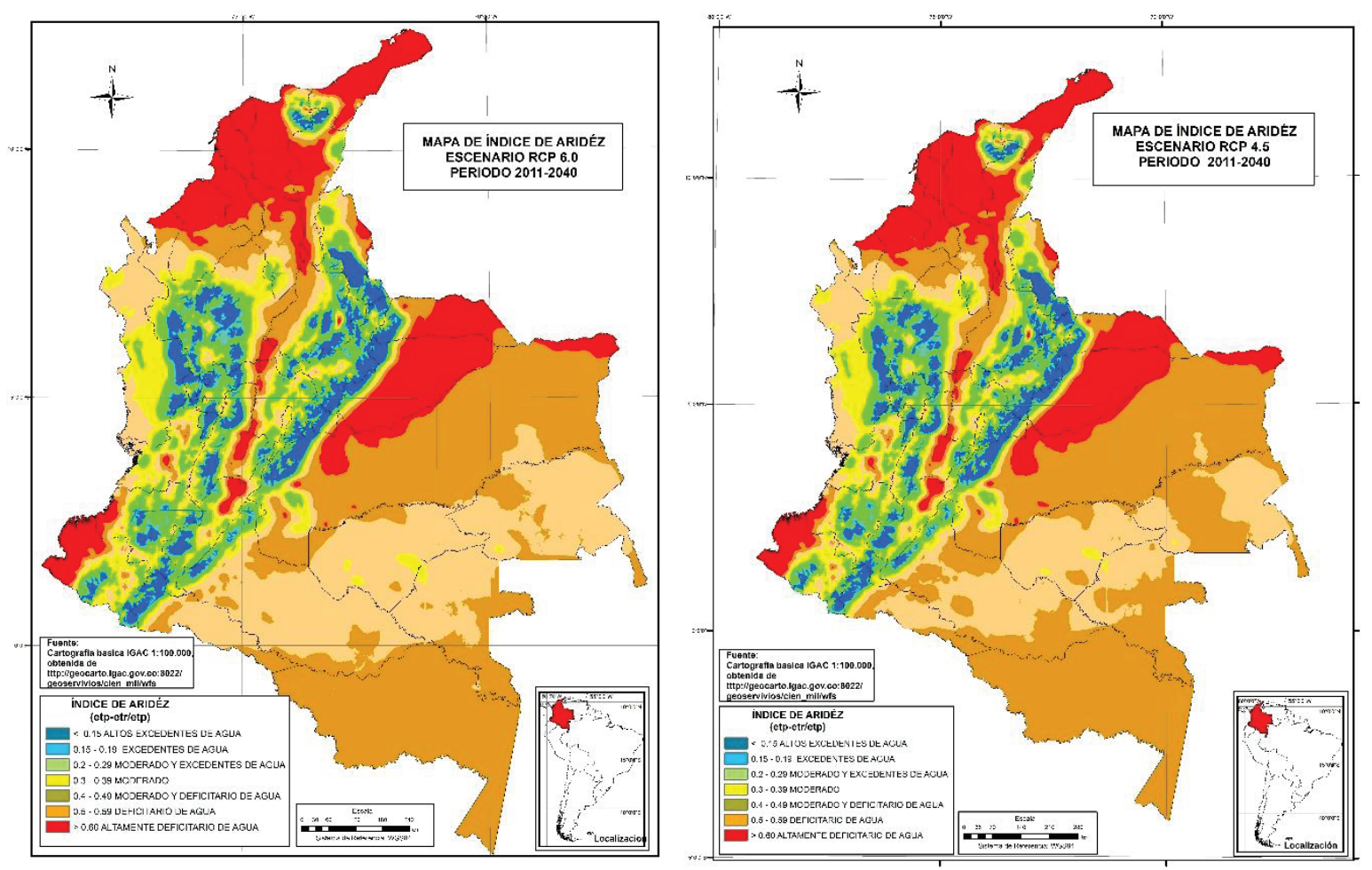

Figura 5. Distribución potencial del índice de aridez bajo los escenarios 6,0 y 4,5, periodo 2011-2040.

Los resultados encontrados con los dos escenarios usados (6,0 y $4,5)$ concuerdan, en gran medida, con las tendencias planetarias esperadas: Colombia es un país altamente vulnerable a los posibles impactos del cambio climático y el régimen hidrológico del país se podría ver altamente afectado, en términos de escorrentía promedio, hasta en un $15 \%$, con relación a la actual. La reducción más drástica se podría presentar en el escenario 6,0, hasta en un $18 \%$, respecto a la condición actual.
Con relación a las condiciones de aridez, según esta modelación, el país pasaría de un índice de aridez moderado y con excedentes de agua a una condición deficitaria de agua, para el escenario 6,0, siendo este el más agresivo. El escenario 4,5 dejaría al país en una condición moderada a deficitaria de agua, siendo, de todas maneras, una condición desfavorable con relación a los promedios actuales, como se puede ver en la tabla 1. Los territorios con moderados, excedentes y altos excedentes de agua en los escenarios modelados

Tabla 1. Índices de aridez futuros bajos los escenarios RCP 6, y 4,5.

\begin{tabular}{|l|c|c|c|}
\hline \multicolumn{1}{|c|}{ ZONA } & \multirow{2}{*}{ ACTUAL } & RCP 6,0 & RCP 4.5 \\
\cline { 3 - 4 } & & $\mathbf{2 0 1 1 - 2 0 4 0}$ & $\mathbf{2 0 1 1 - 2 0 4 1}$ \\
\hline Altos Excedentes de agua & $21,6 \%$ & $5,9 \%$ & $2,7 \%$ \\
\hline Excedentes de agua & $20,2 \%$ & $2,8 \%$ & $1,6 \%$ \\
\hline Moderado y excedentes de agua & $27,1 \%$ & $8,3 \%$ & $5,4 \%$ \\
\hline Moderado & $11,3 \%$ & $8,9 \%$ & $8,1 \%$ \\
\hline Moderado y deficitario de agua & $10,1 \%$ & $26,0 \%$ & $30,3 \%$ \\
\hline Deficitario de agua & $6,9 \%$ & $33,3 \%$ & $32,8 \%$ \\
\hline Altamente deficitario de agua & $2,9 \%$ & $14,8 \%$ & $19,1 \%$ \\
\hline
\end{tabular}


tienden a la baja, mientas que las áreas con condiciones moderadas, deficitarias y altamente deficitarias de agua tienden a incrementarse en todo el territorio colombiano, situación muy compleja, debido a la estrecha relación entre los recursos hídricos y las actividades socioeconómicas del país. Las áreas altamente deficitarias de agua se incrementarían de manera considerable, ubicándose, principalmente, en la Costa Caribe, el valle del río Magdalena y la Orinoquia colombiana; asimismo, los territorios con altos excedentes de agua tienden a reducirse notoriamente, pasando a ser territorios moderados y deficitarios de agua.

Los resultados encontrados en esta investigación sugieren que el cambio climático podría generar efectos negativos sobre los recursos hídricos en el territorio nacional; así, la cuantificación espacio temporal de dichos impactos será de vital importancia en aras de generar políticas, que permitan dar respuestas inmediatas a las posibles afectaciones del recurso, asociadas con este cambio y deben servir de base para una correcta gestión y uso del agua y una ocupación del territorio con una visión de largo plazo; en síntesis, planificar el recurso agua es parte de los retos que genera el cambio climático.

Conflictos de intereses: El manuscrito fue preparado y revisado con la participación de todos los autores, quienes declaramos que no existe ningún conflicto de intereses que ponga en riesgo la validez de los resultados presentados.

\section{REFERENCIAS}

1. AGRAWALA, S.; FANKHAUSER, S. 2008. Economic Aspects of Adaptation to Climate Change. Costs, Benefits and Policy Instruments, París: OCDE. 133p.

2. ALARCÓN, J.; PABÓN, J. 2013. El cambio climático y la distribución espacial de las formaciones vegetales en Colombia. Colombia Forestal. 16(2):171-181.

3. DÖLL, P.; KASPAR, F.; ALCAMO, J. 1999. Computation of global water availabitily and water use at the scale of large drainage basins. Mathematische Geologie. 4:111-118.

4. DRUMMOND, J.; BILLEN, R.; JOAO, E.; FORREST, D. 2007. Dynamic and Mobile GIS: Investigating Changes in Space and Time. CRC Press, Taylor and Francis Group, New York. 344p.

5. ESTRELA, T.; QUINTAS, L. 1996. El sistema integrado de modelización precipitación escorrentía (SIMPA). Revista de Ingeniería Civil- CEDEX. 104:43-52.

6. ESTUDIO NACIONAL DEL AGUA, ENA. 2014. Instituto de Hidrología, Meteorología y Estudios Ambientales. Bogotá D.C. $496 \mathrm{p}$.

7. GUO, S.; WANG, J.; XIONG, L.; YING, Y.; LI, D. 2002. A macro-scale and semi-distributed monthly water balance model to predict climate change impacts in China. J. Hydrology. 268(1):1-15.

8. HENDRA, P.; YASUHIRO, M.; HIROI, K.; IBRAHIM, D.; ATSUSH, M. 2013. Development of Water Management Modeling by using GIS in Chirchik River Basin, Uzbekistan. Procedia Earth and Planetary Science. 6:169-176. https:// doi.org/10.1016/j.proeps.2013.01.023

9. IDEAM, PNUD, MADS, DNP, CANCILLERÍA. 2015. Nuevos Escenarios de Cambio Climático para Colombia 2011 - 2100. Herramientas Científicas para la Toma de Decisiones - Enfoque Nacional - Departamental: Tercera Comunicación Nacional de Cambio Climático. Bogotá. Disponible desde Internet en: http://documentacion. ideam.gov.co/openbiblio/bvirtual/022964/documento_ nacional_departamental.pdf (con acceso 07/082016).

10. INTERGOVERNMENTAL PANEL ON CLIMATE CHANGE IPCC. 2016. Cambio Climático 2014. Informe de síntesis. Grupo Intergubernamental de Expertos sobre el Cambio Climático. Ginebra, Suiza. 176p.

11. MAGRIN, G.; GAY, C.; CRUZ, J.; GIMÉNEZ, A.; MORENO, G.; NAGY, C.; VILLAMIZAR, A. 2007. Latin America. Climate Change. Impacts, Adaptation and Vulnerability. Contribution of Working Group II to the Fourth Assessment Report of the Intergovernmental Panel on Climate Change. Cambridge, UK. 976p.

12. MENZEL, L.; BURGER, G. 2002. Climate change scenarios and runoff response in the Mulde catchment (southern Elbe, Germany). J. Hydrol. 267(1-2):53-64. https://doi. org/10.1016/S0022-1694(02)00139-7

13. MUZIK, I. 2002. A first-order analysis of the climate change effect on the flood frequencies in a sub-alpine watershed by means of a hydrological rainfall-runoff model. J. Hydrol. 267(1-2):65-73. https://doi.org/10.1016/S00221694(02)00140-3

14. TIAN, Y.; WEN, CH.; HONG, S. 2008. Global scientific production on GIS research by bibliometric analysis from 1997 to 2006. J. Informetrics. 2(1):65-74. https://doi. org/10.1016/j.joi.2007.10.001

15. TURC, L. 1954. Le bilan d'eau des sols. Relation entre la precipitation, l'évaporation et l'écoulement. Ann. Agron. 5:491-569.

16. UNESCO. 2010. Glosario hidrológico internacional. Disponible desde Internet en: https:/ / unesdoc.unesco.org/ ark:/48223/pf0000221862 (con acceso el 21/08/2014). 
17. YATES, D.N. 1997. Approaches to continental scale runoff for integrated assessment models. J. Hydrology 201:289-310. https://doi.org/10.1016/S0022-1694(97)00044-9

18. VÖRÖSMARTY, C.J.; FEDERER, C.A.; SCHLOSS, A. 1998. Potential evaporation functions compared on US watersheds: implications for global-scale water balance and terrestrial ecosystem modeling. J. Hydrol. 207(3-4):147-169. https://doi.org/10.1016/S0022-1694(98)00109-7
19. YANG, Y.; FENG, Z.; HUANG, H.; LIN, Y. 2008. Climateinduced changes in crop water balance during 1960-2001 in Northwest China Agriculture. Ecosystems \& Environment. $127(2): 107-118$. http://dx.doi.org/10.1016/j. agee.2008.03.007 